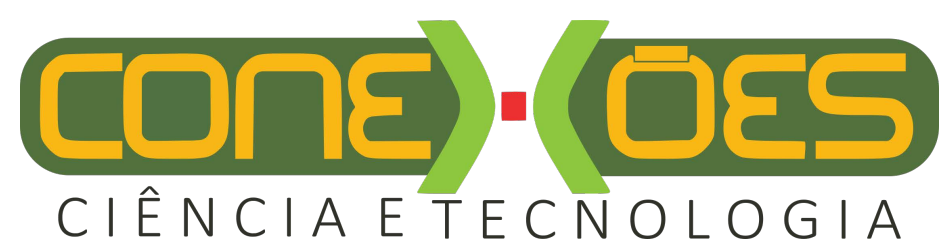

\title{
AVANÇO DO PERIÉLIO DE MERCÚRIO - O PRIMEIRO SUCESSO DA TEORIA DA RELATIVIDADE GERAL DE EINSTEIN
}

\author{
OSWALDO DUARTE MIRANDA \\ Instituto Nacional de Pesquisas Espaciais (INPE) \\ São José dos Campos - SP \\ <oswaldo.miranda@inpe.br>
}

DOI: $10.21439 /$ conexoes.v13i2.1670

\begin{abstract}
Resumo. Em conjunto com o desvio da luz de estrelas distantes pelo campo gravitacional do Sol (comprovado através de observações durante o eclipse solar de 1919), e do desvio para o vermelho na frequência de um feixe de luz pela ação de um campo gravitacional (comprovado pelo experimento de Pound e Rebka realizado em 1960), o movimento secular do periélio de Mercúrio configura-se num dos chamados "testes clássicos" da teoria da relatividade geral (TRG) de Albert Einstein. Em particular, o cálculo do movimento anômalo de Mercúrio foi a primeira peça de evidência empírica que ajudou a estabelecer a TRG como uma das mais belas teorias da física. Em 1915, Einstein incluiu esse cálculo, como uma aplicação, no artigo em que apresentou a sua teoria de gravitação. O valor obtido através da TRG contribuiu para dar confiança à nova teoria através de um problema empiricamente testável e que, na sua primeira aplicação, resolvia um dos maiores problemas da mecânica celeste à época. Neste trabalho, descrevemos os aspectos históricos relacionados aos estudos sobre os movimentos dos planetas. Em particular, discutimos como o movimento anômalo de Mercúrio foi analisado no âmbito da mecânica clássica, comparando a previsão clássica com os dados observacionais estabelecidos naquele período. Finalmente, comparamos a resposta clássica ao movimento anômalo de Mercúrio com o valor obtido através da TRG - o primeiro sucesso da teoria de gravitação de Einstein.
\end{abstract}

Palavras-chaves: Teoria da Relatividade Geral. Mecânica Celeste. Avanço no Periélio de Mercúrio.

\begin{abstract}
Together with the deflexion of the light from distant stars by the gravitational field of the Sun (as evidenced by observations during the solar eclipse of 1919), and the redshift in the frequency of a light beam by the action of a gravitational field (as verified by the experiment carried out by Pound and Rebka in 1960), the secular motion of the perihelion of Mercury is one of the so-called "classical tests" of Albert Einstein's theory of general relativity (TGR). In particular, the calculation of the anomalous motion of Mercury was the first piece of empirical evidence to help establish TGR as one of the most beautiful theories of physics. In 1915, Einstein included this calculation as an application in the article in which he presented his theory of gravitation. The value obtained via TGR contributed to give confidence to the new theory through an empirically testable problem and that, in its first application, solved one of the major problems of celestial mechanics. In this work, we describe the historical aspects related to the studies on the motions of planets. In particular, we discuss how the anomalous motion of Mercury was analyzed in the scope of classical mechanics comparing the classical prediction with the observational data established at that time. Finally, we compare the classical response to the anomalous motion of Mercury with the value obtained through TGR - the first success of the Einstein's gravitation theory.
\end{abstract}

Keywords: Theory of General Relativity. Celestial Mechanics. Perihelion of Mercury Advance.

\section{INTRODUÇÃO}

Historicamente, os primeiros estudos relacionados ao movimento planetário têm suas raízes na antiga Me- sopotâmia, região hoje compreendida pelo Iraque, em sua totalidade, e partes da Síria, Irã, Líbano e Turquia. Tábuas de argila com registros em escrita cuneiforme 


\section{CORE) ( (OES \\ CIÊNCIAETECNOLOGIA}

AVANÇO DO PERIÉLIO DE MERCÚRIO - O PRIMEIRO SUCESSO DA TEORIA DA RELATIVIDADE GERAL DE EINSTEIN

de 2.000 a.C. revelam um rico conhecimento astronômico pelos povos que habitaram essa região (NEUGEBAUER, 1975; NEUGEBAUER, 1983, LAMBERT, 1987; EVANS, 1998). As 12 constelações zodiacais, chamadas de "rebanho brilhante" por esses povos, observações de estrelas e dos planetas Mercúrio, Vênus, Marte, Júpiter e Saturno estão registradas em tábuas de argila produzidas na "Baixa Mesopotâmia" (Suméria) dentro desse período.

Com a consolidação do império grego estendendose até o mar Negro, por volta de $\mathrm{V}$ a.C., é provável que o conhecimento astronômico da Mesopotâmia tenha sido absorvido pela Grécia antiga. Eudoxo de Cnido, que viveu na Ásia Menor no século IV a.C., deixou registros que mostram a importância dos "astrônomos babilônios" sobre o seu trabalho em astronomia. Eudoxo, além de brilhante matemático, concentrou-se principalmente no estudo dos movimentos aparentes dos planetas.

Os planetas mais afastados se movem de leste para oeste, mas ocasionalmente parecem retroceder movendo-se de oeste para leste. Eudoxo acreditava no modelo Geocêntrico, de forma que idealizou um sofisticado esquema para explicar essas irregularidades nos movimentos dos planetas e da Lua. O termo "planetas" vem do grego $\pi \lambda \boldsymbol{\lambda} \boldsymbol{\nu} \eta \boldsymbol{\tau} \boldsymbol{\alpha} \iota$ que significa "estrelas indisciplinadas" (ou errantes).

Por volta do ano 280 a.C. Aristarco, nascido na ilha de Samos no mar Egeu Oriental, foi o primeiro astrônomo a propor que a Terra girava ao redor do Sol (modelo Heliocêntrico). Contudo, suas ideias foram rejeitadas em favor do modelo Geocêntrico. A concepção de Aristarco somente foi retomada depois de decorridos dezoito séculos, em plena idade média.

No século XIII, os trabalhos dos astrônomos gregos chegaram ao mundo ocidental através de traduções preservadas pelos árabes (NEUGEBAUER, 1975, NEUGEBAUER, 1983).

Um jovem astrônomo polonês de nome Nicolau Copérnico identificou, a partir dessas traduções, que o modelo mais simples e elegante, para explicar os movimentos dos planetas, consistia em que estes descrevessem órbitas circulares ao redor do Sol. No ano da sua morte, em 1543, as suas conclusões foram publicadas no livro De revolutionibus orbium coelestium.

A contribuição seguinte ao estudo dos movimentos planetários foi dada pelas numerosas observações realizadas pelo astrônomo dinamarquês Tycho Brahe, nascido três anos após a publicação do livro de Copérnico.

Tycho não aceitava completamente o modelo de Copérnico. Corretamente, ele considerava que a Lua orbitava a Terra e que os planetas orbitavam o Sol. Contudo, erroneamente, seu sistema considerava o Sol orbitando a Terra.

Porém, as observações que ele realizou, ao longo de vários anos, permitiram ao seu assistente, chamado Johannes Kepler, concluir que o modelo de Copérnico se ajustava muito bem aos dados observacionais desde que as órbitas planetárias circulares fossem substituídas por elipticas.

As conclusões de Kepler podem ser resumidas em suas três bem conhecidas leis do movimento planetário:

- A órbita de um planeta é uma elipse com o Sol em um dos seus focos;

- A linha que une o Sol a um planeta varre áreas iguais em intervalos iguais de tempo, independentemente do comprimento da linha;

- O quadrado do período $(P)$ de qualquer planeta é proporcional ao cubo da sua distância média $(R)$ ao Sol, ou seja, $P^{2}=k R^{3}$. A constante $k$ é a mesma para todos os planetas.

Com as leis de Kepler, originalmente publicadas no período 1609 a 1619, a teoria Heliocêntrica passou a ser vista como um modelo de funcionamento do sistema solar.

Por outro lado, Galileo Galilei, nascido em 1564, foi fundamental para o avanço do método científico, tanto em métodos de observação quanto de experimentação. Embora Galileo não tenha inventado o telescópio, ele talvez tenha sido o primeiro a fazer uso sistemático desse instrumento para observações.

Através do telescópio, ele identificou as quatro grandes luas de Júpiter. Isso possibilitou fazer uma analogia direta entre o sistema Terra-Lua com outros corpos do sistema solar. Galileo também demonstrou que as diferentes fases do planeta Vênus poderiam ser explicadas se esse planeta girasse ao redor do Sol.

Isaac Newton, nascido um ano após a morte de Galileo Galilei (ocorrida em 1642), trouxe uma nova percepção sobre os movimentos planetários. Newton demonstrou que a força de atração gravitacional entre dois corpos varia de forma inversamente proporcional ao quadrado da distância de separação e é diretamente proporcional ao produto das massas dos corpos envolvidos, que hoje chamamos de lei da gravitação de Newton.

O seu livro, cujo título original é Philosophiae $\mathrm{Na}$ turalis Principia Mathematica, publicado em 1687, é um marco extraordinário em ciências naturais. Contém as leis para o movimento dos corpos, a fundamentação da mecânica clássica, assim como a lei da gravitação 


\section{CORE) ( (OES \\ CI ÊNCIAETECNOLOGIA}

AVANÇO DO PERIÉLIO DE MERCÚRIO - O PRIMEIRO SUCESSO DA TEORIA DA RELATIVIDADE GERAL DE EINSTEIN

\section{universal 1}

Para poder demonstrar que a força de atração gravitacional, entre dois corpos, poderia ser determinada considerando que a massa de cada corpo concentravase num ponto central, Newton precisou desenvolver um ramo inteiramente novo da matemática, que hoje chamamos de cálculo diferencial e integral.

Newton generalizou as hipóteses da força gravitacional, explicando as leis de Kepler do movimento planetário, bem como explicando através da sua lei "universal" da gravitação como pares de partículas interagiam no Universo.

No século XVIII, o astrônomo William Herschel descobriu que sistemas binários, isto é, "estrelas duplas" em que uma estrela orbita o redor da outra, obedeciam à lei de gravitação de Newton, da mesma forma que os planetas do sistema solar. Herschel tornou-se um astrônomo famoso por ter descoberto o planeta Urano em 1781

Ele buscava identificar sistemas estelares binários, mas no dia 13 de março, Herschel identificou um pequeno ponto luminoso no céu, que inicialmente parecia um cometa. Após acompanhar o objeto por algumas semanas, ele conseguiu identificar que tratava-se de um planeta - Urano - com órbita mais distante que Saturno. O sistema solar ganhava assim mais um planeta em relação aos que eram conhecidos desde a Mesopotâmia.

No início do século XIX, existiam diferentes métodos matemáticos para previsão dos movimentos dos planetas. Estes haviam sido desenvolvidos ao longo de várias décadas por diferentes astrônomos. Em 1839 , o astrônomo francês Urbain-Jean-Joseph Le Verrier concentrou-se no cálculo preciso das órbitas planetárias.

Mercúrio é o planeta mais próximo do Sol, possui curto período de translação e elevada excentricidade de órbita. Isso fez com que Le Verrier começasse, por volta de 1841, a analisar com mais atenção a órbita desse planeta, procurando determiná-la com precisão.

Os seus primeiros trabalhos sobre o cálculo da órbita de Mercúrio foram publicados em 1843 mas com um resultado que o próprio Le Verrier considerava insatisfatório (LE-VERRIER, 1843a; LE-VERRIER 1843b)

Le Verrier deixou Mercúrio de lado e passou a concentrar a atenção sobre Urano. Ele trabalhou durante vários meses em complexos cálculos para explicar as pequenas discrepâncias entre a órbita observada de Urano e aquela prevista pelas leis de Newton. Em

${ }^{1}$ Recomendo aos interessados a versão em língua portuguesa publicada pela Editora da Universidade de São Paulo (NEWTON 2008a NEWTON 2008b.
31 de agosto de 1846, Le Verrier apresentou sua análise final à Academia Francesa de Ciências, prevendo que as pequenas discrepâncias na órbita de Urano eram ocasionadas por um "planeta invisível".

Ele encaminhou carta a Johann Galle, do Observatório de Berlim, com a órbita prevista para o "novo planeta". A carta chegou em 23 de setembro de 1846 e na mesma noite Galle e o astrônomo Heinrich d'Arrest encontraram o "planeta de Le Verrier" - Netuno - dentro da área prevista por esse astrônomo 2

Com o sucesso alcançado a partir da descoberta de Netuno, Le Verrier assumiu a tarefa de colocar em harmonia todo o sistema planetário. Caso não fosse possível explicar com precisão os dados observacionais, então ainda existiriam causas desconhecidas de perturbações gravitacionais (LéVY, 1968).

Ele começou então por reavaliar, até a $7 \underline{a}$ ordem, as perturbações planetárias conhecidas. Essa derivação, que resultou em 469 termos matemáticos, foi concluída em 1849. Em seguida, ele coletou observações das posições dos planetas (dados observacionais de 1750), avaliando-os e corrigindo as inconsistências com os dados mais recentes. Isso o ocupou pelos anos seguintes.

Em 1859, Le Verrier retoma o estudo sobre o movimento de Mercúrio. Examinando os registros dos trânsitos desse planeta, ele concluiu que a órbita precessionava lentamente. Em princípio, resultado natural se a influência dos demais planetas fosse levada em conta.

A precessão observada era de 565 segundos de arco por século. Le Verrier obteve a partir dos seus cálculos o valor de 526,7 segundos de arco por século.

Essa diferença de 38,3 " por século 3 entre os dois valores, aparentemente, não podia ser absorvida pela teoria Newtoniana de gravitação através dos métodos perturbativos desenvolvidos por Le Verrier.

Os incrementos esperados para a órbita de Mercúrio, a partir de cada um dos planetas que Le Verrier utilizou em seus cálculos, forneciam por século:

- Vênus: 280, 6"

- Terra: $83,6 "$

- Marte: 2,6"

- Júpiter: $152,6 "$

- Saturno: 7,2 "

- Urano: $0,1 "$

\footnotetext{
${ }^{2} \mathrm{O}$ inglês John Couch Adams trabalhou de forma independente de Le Verrier sobre as discrepâncias na órbita de Urano. Adams encaminhou sua solução ao Royal Greenwich Observatory dois dias após a comunicação de Le Verrier à Academia Francesa.

${ }^{3} \mathrm{O}$ símbolo " representa segundos de arco.
} 


\section{CORE) ( (OES \\ CI ÊNCIAETECNOLOGIA}

AVANÇO DO PERIÉLIO DE MERCÚRIO - O PRIMEIRO SUCESSO DA TEORIA DA RELATIVIDADE GERAL DE EINSTEIN

Vênus, vizinho mais próximo de Mercúrio, é o principal culpado pelo movimento anômalo, mas os outros planetas também contribuem em maior ou menor grau. Note que Júpiter, planeta mais massivo do sistema solar, contribui com quase 30 por cento da taxa de avanço do periélio de Mercúrio, apesar de sua grande distância do Sol.

As perturbações planetárias levam a uma taxa de avanço do periélio de Mercúrio de 1,27" por órbita ou, aproximadamente, 527 " por século 4

Em função dessa diferença de 38, 3" e motivado pelo sucesso da descoberta de Netuno, Le Verrier inferiu que um aumento de aproximadamente 10 por cento na massa de Vênus explicaria o avanço do periélio de Mercúrio, mas esse incremento de massa também afetaria a órbita da Terra de uma maneira que não havia sido observada até então.

Como a "massa faltante" não deveria afetar a órbita da Terra, Le Verrier inferiu que ela deveria estar mais perto do Sol do que a órbita de Mercúrio. Assim começou a busca ao "novo planeta invisível" de Le Verrier, ou melhor, aos "planetas invisíveis".

Ele rapidamente percebeu que um único planeta tão perto do Sol teria um enorme brilho e, portanto, seria visível durante os eclipses solares. Como nenhum planeta desse tipo havia sido observado durante eclipses passados, Le Verrier supôs que a massa estaria na forma de muitos corpos pequenos.

Contudo, em dezembro de 1859, Le Verrier recebeu uma surpreendente comunicação do médico francês, e astrônomo amador, Edmond Modeste Lescarbault da vila de Orgères-en-beauce na França. Lescarbault informava que havia registrado observações durante o que ele acreditava ser um trânsito de um "planeta intermercurial".

Le Verrier, convencido pela história, divulgou a notícia do novo planeta, que foi rapidamente denominado de Vulcano - o deus do fogo na mitologia romana. Ele havia, novamente, encantado a comunidade científica francesa. A dupla formada por Isaac Newton e UrbainJean-Joseph Le Verrier novamente triunfou, ao menos era o que parecia.

Com base nas observações de Lescarbault, Le Verrier calculou a distância do planeta em relação ao Sol, obtendo 0,147 unidades astronômicas, e determinou seu período como sendo 19 dias e 17 horas (BAUM; SHEEHAN, 1997). A comunidade astronômica tentou re-

\footnotetext{
${ }^{4}$ Mercúrio completa uma órbita ao redor do Sol em, aproximadamente, 88 dias. Um ano terrestre equivale a 365,26 dias de forma que em 100 anos temos 36.526 dias. O número de órbitas de Mercúrio em 100 anos é $36.526 / 88 \approx 415$. Assim, a cada órbita Mercúrio avança seu periélio em $527 / 415 \approx 1,27$ ", conforme a estimativa de Le Verrier.
}

petidamente observar o evasivo planeta Vulcano, mas, com o passar do tempo, sem nenhum avistamento, começaram a surgir dúvidas quanto à existência desse planeta.

Com tanta atenção direcionada para observar a área ao redor do Sol durante os eclipses subsequentes, e sem ao menos uma observação positiva, a grande maioria dos astrônomos deixou de acreditar na existência de Vulcano.

Até sua morte, em 1877, Le Verrier permaneceu totalmente convencido de que a "massa faltante" existia e que eventualmente seria encontrada, mostrando mais uma vez a supremacia da lei da gravitação de Newton 5

Em 1882, o astrônomo Simon Newcomb corrigiu algumas inconsistências na massa planetária e repetiu os cálculos de Le Verrier. Ele descobriu um deslocamento extra no periélio de Mercúrio de 43" por século (NEWCOMB, 1882), um pouco maior que o resultado anteriormente obtido. Os dados observacionais analisados por Newcomb mostravam que a precessão de Mercúrio era de 574, 83" por século (NEWCOMB, 1898).

Como a "massa faltante" estava, a essa altura, fora de questão, ele pensou que o problema poderia estar na lei da gravitação de Newton. Newcomb ponderou que se o expoente, na lei do inverso do quadrado da distância, fosse 2,00000016 ao invés de 2, então o movimento de Mercúrio poderia ser explicado com maior precisão.

O raciocínio de Newcomb representou uma verdadeira mudança de paradigma. Enquanto, anteriormente, as observações eram questionadas e a teoria de Newton era inatacável, os cientistas ao final do século XIX começavam a questionar as fundações da lei de gravitação clássica.

Do ponto de vista observacional, o problema estava, essencialmente, fechado. Em contraste, o que existiria de "errado" com a lei da gravitação de Newton passou a ser considerado um problema em aberto. Estavam geradas as condições para que surgisse uma nova teoria de gravitação - A Teoria da Relatividade Geral de Einstein.

Na Seção 2 apresento o "cálculo clássico" para o avanço do periélio de Mercúrio, enquanto na Seção 3, apresento os principais conceitos envolvidos com a determinação da anomalia desse planeta no âmbito da TRG de Einstein. A Seção 4 apresenta as considerações finais deste trabalho.

\footnotetext{
${ }^{5}$ Um interessante texto biográfico sobre Urbain-Jean-Joseph Le Verrier pode ser encontrado no artigo de Laskar (2017).
} 


\section{(CORE) (DES \\ CIÊNCIAE TECNOLOGIA}

AVANÇO DO PERIÉLIO DE MERCÚRIO - O PRIMEIRO SUCESSO DA TEORIA DA RELATIVIDADE GERAL DE EINSTEIN

\section{Precessão do Periélio de Mercúrio: Aborda- gem Clássica}

No âmbito da mecânica clássica de Newton é possível, precisamente, modelar a força gravitacional que cada planeta deveria exercer sobre Mercúrio. Uma aproximação que considero bastante elegante, e acessível, foi publicada em 1979 (PRICE; RUSH, 1979).

Os autores substituíram cada um dos planetas externos a Mercúrio por "anéis de massa" , cada um caracterizado por sua respectiva densidade linear de massa uniforme. Como Mercúrio apresenta lenta precessão, quando comparada com as órbitas planetárias até Urano, a abordagem de Price e Rush produz uma estimativa bastante precisa para descrever os efeitos dos planetas externos sobre a órbita de Mercúrio.

Como mencionado acima, cada planeta é substituído por um anel com densidade linear de massa descrita pela seguinte equação:

$$
\lambda_{i}=\frac{M_{i}}{2 \pi R_{i}},
$$

sendo $\lambda_{i}$ a densidade linear de massa do $i$-ésimo planeta a partir do Sol, $M_{i}$ a massa do $i$-ésimo planeta e $R_{i}$ o raio da órbita, que é considerada circular.

O campo gravitacional de cada planeta exterior a Mercúrio é aproximado como um anel circular, e uniforme, centrado no Sol e no plano definido pela órbita de Mercúrio, conforme Figura 1. Um ponto de massa $m$ está colocado sobre a linha $A B C$ e a distância $a$ do centro $C$ do anel de raio $R$.

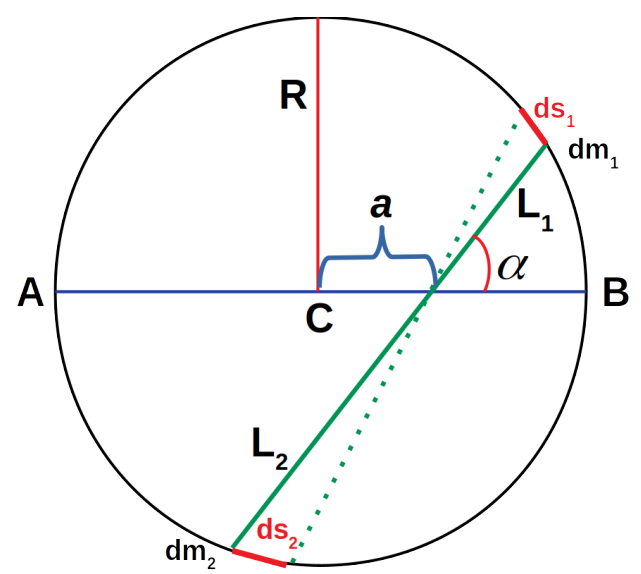

Figura 1: Forma como as forças gravitacionais são calculadas sobre a massa $m$, que está situada à distância $a$ do centro de um anel com densidade linear uniforme de massa $\left(\lambda_{i}\right)$. Figura adaptada pelo autor a partir da original publicada em Price e Rush (1979).

O anel é dividido em elementos diferenciais de massa. Em particular, a Figura 1 mostra os elementos $d m_{1}$ e $d m_{2}$ que estão localizados num ângulo $\alpha$ da linha $A B$. Considere $d s_{1}$ e $d s_{2}$ os arcos subentendidos pelo elemento diferencial angular $d \alpha$ e que estão, respectivamente, associados com os elementos de massa $d m_{1}$ e $d m_{2}$. Então podemos escrever, se $a<<R$

$$
d m_{i}=\lambda d s_{i} \simeq \lambda l_{i} d \alpha,
$$

onde $l_{i}$ é a distância de $m$ ao anel (vide Figura 1 ) e $i$ pode assumir os valores 1 ou 2 .

A lei da gravitação de Newton permite então escrever

$$
d \mathbf{F}=G m\left(\frac{d m_{1}}{l_{1}^{2}}-\frac{d m_{2}}{l_{2}^{2}}\right) \hat{\mathbf{l}},
$$

sendo î um vetor unitário ligando $m$ a $d m_{1}$.

Substituindo a Equação 2 na 3 vem

$$
d \mathbf{F}=G m \lambda\left(\frac{l_{2}-l_{1}}{l_{1} l_{2}}\right) \hat{\mathbf{l}} d \alpha .
$$

Por simetria, os únicos componentes de $d \mathbf{F}$ que afetam $m$ são os que estão sobre a linha $A B$. Os componentes perpendiculares de $d \mathbf{F}$ se cancelam. Dessa forma, atuará sobre $m$ um elemento diferencial de força $d \mathbf{F}_{r}$ dado por

$$
d \mathbf{F}_{r}=d \mathbf{F} \cos \alpha .
$$

Se $\hat{\mathbf{r}}$ é um vetor unitário na direção radial, nós podemos integrar a Equação 5 para obter

$$
\mathbf{F}=\hat{\mathbf{r}} \int_{-\pi / 2}^{+\pi / 2} G m \lambda\left(\frac{l_{2}-l_{1}}{l_{1} l_{2}}\right) \cos \alpha d \alpha,
$$

sendo que os valores escolhidos para os limites de integração permitem cobrir todo o anel.

Através da lei dos cossenos torna-se possível relacionar $\alpha \operatorname{com} l_{1}$ e $l_{2}$. Em particular,

$$
R^{2}=a^{2}+l_{1}^{2}-2 a l_{1} \cos (\pi-\alpha) .
$$

Essa equação quadrática permite obter a solução para $l_{1}$ como

$$
l_{1}=-a \cos \alpha+\left[a^{2} \cos ^{2} \alpha-\left(a^{2}-R^{2}\right)\right]^{1 / 2} .
$$

A escolha do sinal para a raiz da Equação 8 é a que satisfaz o requerimento físico de que para $\alpha=0$ tenha$\operatorname{mos} l_{1}=R-a$ (vide Figura 1 ).

Nós podemos repetir o processo de forma a obter para $l_{2}$

$$
l_{2}=a \cos \alpha+\left[a^{2} \cos ^{2} \alpha-\left(a^{2}-R^{2}\right)\right]^{1 / 2},
$$

onde a Equação 9 permite obter $l_{2}=R+a$ quando $\alpha=0$. 


\section{CORE) (DES \\ CI ÊNCIAE TECNOLOGIA}

AVANÇO DO PERIÉLIO DE MERCÚRIO - O PRIMEIRO SUCESSO DA TEORIA DA RELATIVIDADE GERAL DE EINSTEIN

Substituindo as Equações 8 e 9 na Equação 6 vem

$$
\mathbf{F}=\frac{2 G m a \lambda}{R^{2}-a^{2}} \hat{\mathbf{r}} \int_{-\pi / 2}^{+\pi / 2} \cos ^{2} \alpha d \alpha,
$$

que nos permite então escrever

$$
\mathbf{F}_{i}=\left(\frac{G \pi m a \lambda_{i}}{R_{i}^{2}-a^{2}}\right) \hat{\mathbf{r}} .
$$

A Equação 11 permite determinar a força radial exercida sobre Mercúrio pelo $i$-ésimo planeta. $\mathrm{O}$ parâmetro $a$ fornece a distância de Mercúrio ao Sol, enquanto $\hat{\mathbf{r}}$ é um vetor unitário para a posição de Mercúrio.

Como $R_{i}>a, \mathbf{F}_{i}$ em 11 é positivo, de forma que a força exercida sobre Mercúrio, por cada planeta externo, é direcionada para fora, oposta à força exercida pelo Sol.

Agora, vamos nos referir a alguns resultados da mecânica clássica, considerando que as órbitas dos planetas sejam estáveis e fechadas. Sabemos que o momentum angular $\mathbf{J}$ de uma massa $m$, que se move em relação a um ponto $O$, é definido por

$$
\mathbf{J}=\mathbf{r} \times \mathbf{p},
$$

onde o símbolo $\times$ representa o produto vetorial, $\mathbf{p}=$ $m \dot{\mathbf{r}}=m \mathbf{v}$ é o momentum linear de $m$ e o ponto representa dervivada com relação ao tempo (isto é, $\dot{\mathbf{r}}=$ $d \mathbf{r} / d t=\mathbf{v})$. Veja que $\mathbf{v}$ é a velocidade orbital de $m$.

A evolução no tempo do momentum angular pode ser obtida através de

$$
\dot{\mathbf{J}}=\dot{\mathbf{r}} \times \mathbf{p}+\mathbf{r} \times \dot{\mathbf{p}} .
$$

O primeiro termo do lado direito da Equação 13 é igual a zero, visto que $\dot{\mathbf{r}}=\mathbf{v}$. Isto é, os vetores $\dot{\mathbf{r}}$ e $\mathbf{p}$ estão no mesmo plano, o que faz com que o produto vetorial deles seja igual a zero.

Por outro lado, fazendo uso da segunda lei de Newton, na forma $\mathbf{F}=\dot{\mathbf{p}}$, na Equação 13 vem

$$
\dot{\mathbf{J}}=\mathbf{r} \times \mathbf{F}(\mathbf{r})=\mathbf{r} \times \Phi(r) \hat{\mathbf{r}},
$$

onde $\Phi(r)$ é o módulo da força total aplicada sobre $m$.

Como $\mathbf{r}=r \hat{\mathbf{r}}$ então a Equação 14 retorna $\dot{\mathbf{J}}=0$. Assim, J é uma constante do movimento em relação ao ponto central $O$, significando que o movimento de $m$ por ação da força $\Phi(r)$ ocorre sempre no mesmo plano. Ou seja, o plano da órbita não muda a medida que o planeta orbita o Sol.

Visto que, o movimento orbital ocorre num plano perpendicular ao vetor constante $\mathbf{J}$, torna-se mais conveniente usarmos coordenadas plano-polares em que

$$
\mathbf{r}=r \hat{\mathbf{r}} \quad \text { e } \quad \mathbf{v}=\dot{r} \hat{\mathbf{r}}+r \dot{\theta} \hat{\boldsymbol{\theta}},
$$

com a equação para a aceleração dada por

$$
\mathbf{a}=\ddot{\mathbf{r}}=\left(\ddot{r}-r \dot{\theta}^{2}\right) \hat{\mathbf{r}}+(r \dot{\theta}+2 \dot{r} \dot{\theta}) \hat{\boldsymbol{\theta}} .
$$

A Figura 2, extraída do capítulo 14.6 de ROGAWSKI e COLIN (2015), apresenta as coordenadas plano-polares para descrever o movimento de um planeta ao redor do Sol.

Ao leitor interessado no aprendizado, e compreensão das diversas aplicações, do cálculo diferencial e integral, recomendo essa referência.

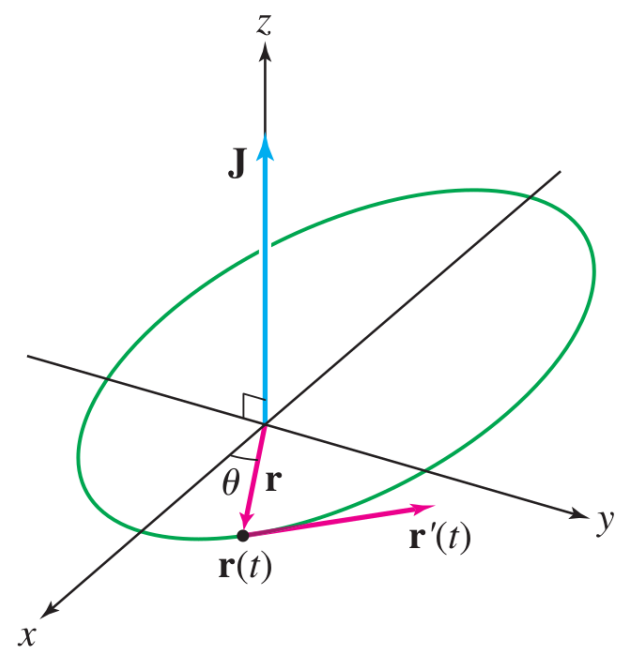

Figura 2: Coordenadas polares $(r, \theta)$ para descrever o movimento orbital de um planeta ao redor do Sol. O deslocamento está contido no plano ortogonal ao vetor momentum angular J. Figura extraída de ROGAWSKI e COLIN (2015). Esses autores representam a derivada em relação ao tempo através do apóstrofo, de forma que $\mathbf{r}^{\prime}(t)=\mathbf{v}=$ $d \mathbf{r} / d t$.

O primeiro termo do lado direito da Equação 16 é o componente radial da aceleração produzida sobre o planeta. Fazendo uso da segunda lei de Newton, podemos escrever

$$
\Phi(r)=m\left(\ddot{r}-r \dot{\theta}^{2}\right) .
$$

Substituindo a Equação 15 na 12, obtemos

$$
\mathbf{J}=\mathbf{r} \times(m \mathbf{v})=m r \hat{\mathbf{r}} \times(\dot{r} \hat{\mathbf{r}}+r \dot{\theta} \hat{\boldsymbol{\theta}}),
$$

que resulta em

$$
\mathbf{J}=m r^{2} \dot{\theta}(\hat{\mathbf{r}} \times \hat{\boldsymbol{\theta}}) .
$$

O produto vetorial de $\hat{\mathbf{r}} \operatorname{com} \hat{\boldsymbol{\theta}}$ fornece a orientação do vetor J. Observe a Figura 2 em que $\hat{\mathbf{r}} \times \hat{\boldsymbol{\theta}}=\hat{\mathbf{z}}$. 


\section{CORE) (DES \\ CIÊNCIAE TECNOLOGIA}

AVANÇO DO PERIÉLIO DE MERCÚRIO - O PRIMEIRO SUCESSO DA TEORIA DA RELATIVIDADE GERAL DE EINSTEIN

Assim, temos para o módulo do vetor $\mathbf{J}$ :

$$
J=m r^{2} \dot{\theta} .
$$
obter

Podemos fazer uso da Equação 20 na 17 de forma a

$$
\Phi(r)=m\left(\ddot{r}-\frac{J^{2}}{m^{2} r^{3}}\right) .
$$

Para o caso particular de órbita circular com raio $a$, temos $\ddot{r}=0$ de forma que a Equação 21 pode ser reescrita como

$$
\Phi(a)=-\frac{J^{2}}{m a^{3}} .
$$

Caso o planeta seja ligeiramente perturbado no plano da sua órbita, e se a perturbação for normal à sua trajetória inicial, ele oscilará ao redor de $a$. Definindo uma nova variável $x \equiv r-a$ e expressando a Equação 21 do movimento radial em função de $r=a+x$, torna-se possível escrever, com auxílio da Equação 22, a seguinte relação

$$
\begin{aligned}
\Phi(x+a) & =m \ddot{x}-J^{2} m^{-1}(x+a)^{-3} \\
& =m \ddot{x}-J^{2} m^{-1} a^{-3}\left(1+\frac{x}{a}\right)^{-3}
\end{aligned}
$$

Observe o lado direito da Equação 23 . Como $x / a<<1$ então nós podemos expandir o termo dentro dos parênteses através de um binômio, retendo apenas os termos de primeira ordem. Por outro lado, $\Phi(x+a)$, do lado esquerdo da igualdade, pode ser expandido em série de Taylor ao redor do ponto $r=a$. Novamente, retemos apenas os termos de primeira ordem 6 Dessa forma, a Equação 23 pode ser reescrita como

$$
\ddot{x}+\left(\frac{1}{m}\right)\left[-\left(\frac{3}{a}\right) \Phi(a)-\Phi^{\prime}(a)\right] x=0,
$$

onde $\Phi^{\prime}(a)=d \Phi / d r$ calculada no ponto $r=a$.

A Equação 24 descreve um oscilador harmônico se o termo dentro dos colchetes for positivo. Se esse termo for negativo, as oscilações crescerão de forma exponencial com o tempo, o que tornará a órbita instável.

A equação de um oscilador harmônico tem a forma geral dada por

$$
\ddot{x}+\omega^{2} x=0,
$$

${ }^{6} \mathrm{O}$ leitor que tenha feito um ano de cálculo diferencial e integral pode verificar os passos intermediários de desenvolvimento. Isto é, como chegar na Equação 24 a partir da 23. De toda forma, recomendo o texto ROGAWSKI e COLIN (2015) em caso de dúvidas. sendo $\omega$ a frequência angular das oscilações, que se relaciona com o período $T$, das oscilações, através de $\omega=2 \pi / T$.

Dessa forma, para órbitas estáveis, podemos obter o período das oscilações ao redor do ponto $r=a$ como

$$
T=2 \pi\left[\frac{m}{-(3 / a) \Phi(a)-\Phi^{\prime}(a)}\right]^{1 / 2} .
$$

Por definição, "apside" é o ponto de maior ou de menor distância, medido a partir do eixo maior de uma elipse, separando um objeto celeste do centro da atração gravitacional (que está colocado num dos focos da elipse).

O ponto mais distante do centro da atração gravitacional é chamado de "apoapside" (ou apocentro), enquanto o ponto mais próximo é chamado de "periapside" (ou pericentro). Quando o centro da atração gravitacional é o Sol, o apocentro passa a se chamar "afélio" , enquanto o pericentro passa a ser chamado de "periélio".

Na Figura 3 pode-se observar o comportamento da órbita de Mercúrio ao redor do Sol. É possível ver a "precessão apsidal" da órbita desse planeta. O ângulo apsidal $(\Psi)$ é o ângulo formado entre duas apsides consecutivas. É a partir desse ângulo que podemos determinar a taxa com que o periélio de Mercúrio precessiona.

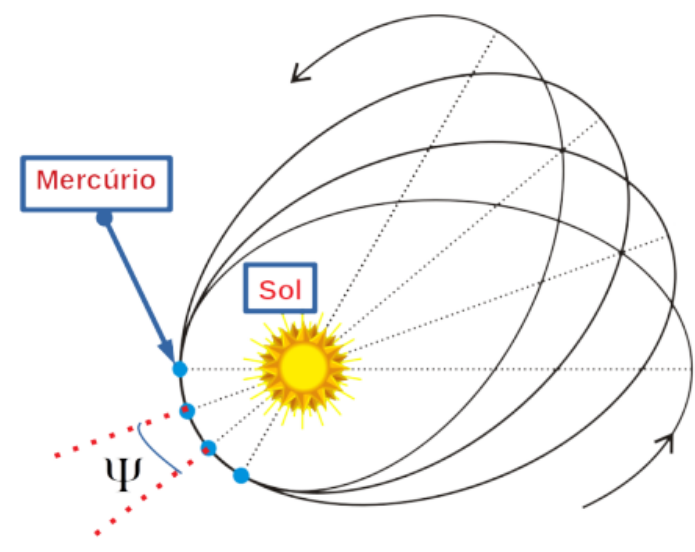

Figura 3: A órbita de Mercúrio ao redor do Sol. Pode-se identificar a precessão apsidal, que é medida através do ângulo $\Psi$. Veja que o eixo maior da elipse, com o Sol num dos focos, permite identificar, para cada uma das órbitas, tanto o afélio quanto o periélio. Figura produzida pelo autor.

O tempo necessário para Mercúrio cobrir o ângulo $\Psi$ é igual a $T / 2$. Como $r$ terá "aproximadamente" o valor $a$, a precessão da órbita com o tempo pode ser 


\section{(CORE). (OES \\ CIÊNCIAETECNOLOGIA}

AVANÇO DO PERIÉLIO DE MERCÚRIO - O PRIMEIRO SUCESSO DA TEORIA DA RELATIVIDADE GERAL DE EINSTEIN

absorvida através de $\dot{\theta}$ (vide Figura 2 ). Assim, com auxílio da Equação 20 podemos escrever $(\operatorname{com} r=a)$

$$
\begin{aligned}
\Psi & =\frac{1}{2} T \dot{\theta} \\
& =\frac{1}{2}\left\{2 \pi\left[\frac{m}{-(3 / a) \Phi(a)-\Phi^{\prime}(a)}\right]^{1 / 2}\right\}\left(\frac{J}{m a^{2}}\right)
\end{aligned}
$$

Por outro lado, usando a Equação 22 na 27 vem

$$
\Psi=\pi\left\{3+a\left[\frac{\Phi^{\prime}(a)}{\Phi(a)}\right]\right\}^{-1 / 2} .
$$

Note que nesse resultado, $\Phi(a)$ é a força central líquida e $a$ é o raio da órbita circular que está sendo perturbada.

Agora, podemos usar a Equação 11 para encontrar a soma das forças de todos os anéis planetários externos a Mercúrio. Isto é,

$$
F(a)=G \pi m \sum_{i=2}^{6} \frac{\lambda_{i} a}{R_{i}^{2}-a^{2}}=7,587 \times 10^{15} N
$$

O valor numérico foi obtido substituindo as massas e os raios orbitais de todos os planetas externos a Mercúrio 7

Note que fizemos uso da Equação 1 que permite relacionar massa e distância orbital de cada planeta e a somatória em 29 refere-se aos planetas Vênus, Terra, Marte, Júpiter e Saturno, de forma que a soma vai de 2 até 6 . $\mathrm{O}$ valor $i=1$ corresponde a Mercúrio.

A força gravitacional exercida pelo Sol sobre Mercúrio é

$$
F_{\odot}(r)=-\frac{G M_{\odot} m}{r^{2}}=-1,318 \times 10^{22} N
$$

onde $M_{\odot}$ representa a massa do Sol.

Assim, a força líquida que Mercúrio experimenta é

$$
\Phi(a)=F_{\odot}+F(a) .
$$

Nós podemos agora determinar o ângulo $\Psi$ na Equação 28. Para isso, precisamos de $\Phi(a)$ e de sua derivada $\Phi^{\prime}(a)$.

\footnotetext{
${ }^{7}$ Os leitores interessados nos valores numéricos, para as massas e raios orbitais dos planetas, poderão encontrar facilmente essas informações no curso, on-line sobre Astronomia e Astrofísica, dos Professores Kepler de Souza Oliveira Filho e Maria de Fátima Oliveira Saraiva do Instituto de Física da Universidade Federal do Rio Grande do Sul (IF-UFRGS). Veja, em particular, o hiperlink $<$ http://astro.if.ufrgs.br/ssolar.htm>
}

Dessa forma, diferenciando a Equação 31 e multiplicando por $a$ vem

$$
a \Phi^{\prime}(a)=a\left[F_{\odot}^{\prime}(a)+F^{\prime}(a)\right]
$$

A derivada da Equação 30 fica

$$
a F_{\odot}^{\prime}(a)=a\left(\frac{2 G M_{\odot} m}{a^{3}}\right)=-2 F_{\odot} .
$$

Quanto à derivada da Equação 29, temos

$$
\begin{aligned}
a F^{\prime}(a) & =G \pi m a \sum_{i=2}^{6} \lambda_{i} \frac{R_{i}^{2}+a^{2}}{\left(R_{i}^{2}-a^{2}\right)^{2}} \\
& =G \pi m a S,
\end{aligned}
$$

onde o termo em somatória foi definido como a função $S$.

Substituindo o conjunto de Equações 29 a 34 na 28 vem

$$
\Psi=\pi\left[3+\frac{G \pi m a S-2 F_{\odot}}{F_{\odot}+F(a)}\right]^{-1 / 2},
$$

que pode ser reescrita como

$$
\Psi=\pi\left[\frac{1+[3 F(a)+G \pi m a S] / F_{\odot}}{1+\left[F(a) / F_{\odot}\right]}\right]^{-1 / 2} .
$$

Podemos fazer uma expansão binomial para o numerador e o denominador da Equação 36 visto que $F(a)<<F_{\odot}$ e $F(a)$ é da mesma ordem que o termo $G \pi m a S$. Assim, obtemos após negligenciar os termos de ordem maior que a primeira da razão $F(a) / F_{\odot}$

$$
\Psi=\pi\left[1-\frac{3 F(a)+G \pi m a S}{2 F_{\odot}}\right]\left[1+\frac{F(a)}{F_{\odot}}\right]
$$

Efetuando a multiplicação e negligenciando os termos de segunda ordem, vem

$$
\Psi=\pi\left(1-\frac{F(a)}{F_{\odot}}-\frac{G \pi m a S}{2 F_{\odot}}\right) .
$$

Fazendo uso das massas e raios orbitais dos planetas, torna-se possível obter $\Psi$ como

$$
\Psi=\pi\left(1+9,884 \times 10^{-7}\right) .
$$

A taxa com que o periélio de um planeta precessiona pode ser obtida diretamente do ângulo apsidal através da equação

$$
\dot{\omega}=\frac{2 \Psi-2 \pi}{P}=\frac{\pi\left(1,977 \times 10^{-6}\right)}{87,969 \text { dias }},
$$




\section{CORE) ( (OES \\ CI ÊNCIAETECNOLOGIA}

AVANÇO DO PERIÉLIO DE MERCÚRIO - O PRIMEIRO SUCESSO DA TEORIA DA RELATIVIDADE GERAL DE EINSTEIN

onde $P$ é o período sideral do planeta (para Mercúrio $P=87,969$ dias $)$.

Após converter para segundos de arco por século, obtemos

$$
\dot{\omega}=531,9 " \text { por século. }
$$

Como mencionado na Seção 1, o valor atual total para a precessão do periélio de Mercúrio é de, aproximadamente, $575 "$ por século (NEWCOMB, 1898). Assim, existe uma diferença (anomalia) de 43 " por século que não pode ser absorvida na teoria Newtoniana.

Contudo, como veremos na Seção 3 , essa "anomalia" pode ser perfeitamente absorvida na TRG de Einstein.

\section{Precessão do Periélio de Mercúrio: Aborda- gem Relativística Geral}

Em 18 de novembro de 1915, Einstein apresentou à Academia Prussiana de Ciências seu trabalho, cujo título é Explicação sobre o Movimento do Periélio de Mercúrio a partir da Teoria da Relatividade Geral.

Einstein relatou, nessa palestra, que o movimento do periélio de Mercúrio poderia ser bem explicado (EINSTEIN, 1915a) pela nova teoria de gravitação que ele havia apresentado poucos dias antes, entre 4 e 11 de novembro de 1915 (EINSTEIN, 1915b; EINSTEIN, 1915c).

A TRG de Einstein entende a gravitação como resultado da "curvatura do espaço-tempo" . A forma mais direta de você entender a gravitação, no contexto da TRG, é imaginar uma cama elástica bem esticada representando o espaço-tempo. Colocando uma esfera densa e massiva no centro da cama elástica, esta se curvará. Isto é, a cama elástica se deformará ao redor da esfera massiva.

Coloque agora uma bolinha nas proximidades dessa esfera massiva. Você observará seu deslocamento em direção a parte mais curvada da cama elástica. É algo parecido a esse exemplo que ocorre com o espaçotempo - o "tecido que permeia todo o Universo" .

Na presença de um objeto massivo, o espaço-tempo se curvará, fazendo com que corpos de menor massa, e até mesmo a própria luz, desloquem-se em direção ao objeto que atuou como fonte da curvatura do espaçotempo (MIRANDA, 2019). É esse efeito que chamamos de força gravitacional (vide Figura 4).

As equações de campo da TRG de Einstein são escritas na forma

$$
R_{\mu \nu}-\frac{1}{2} R g_{\mu \nu}=\frac{8 \pi G}{c^{4}} T_{\mu \nu}
$$

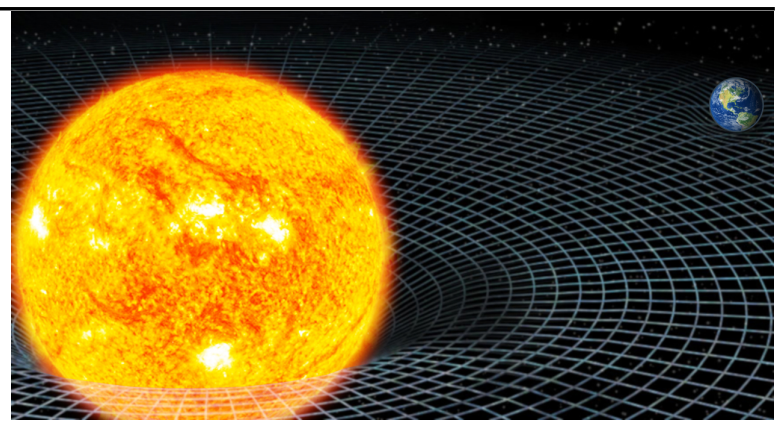

Figura 4: O espaço-tempo sendo curvado pela presença de um objeto massivo (Sol, neste exemplo). Um objeto de menor massa (Terra, neste caso) sente essa curvatura e se desloca descrevendo o movimento orbital. Veja que a Terra também produz curvatura do espaçotempo ao seu redor. Contudo, a deformação é mais acentuada na vizinhança do Sol, por este possuir massa muito maior que a do nosso planeta (MIRANDA 2019) . A Figura foi produzida por Schwarza, Divulgador e Youtuber do Canal Poligonautas.

onde $R_{\mu \nu}$ é o tensor de curvatura de Ricci, $R$ é o escalar de curvatura, $g_{\mu \nu}$ é o tensor métrico, $G$ é a constante de gravitação universal, $c$ é a velocidade da luz no vácuo e $T_{\mu \nu}$ é o tensor energia-momentum.

Os índices $\mu$ e $\nu$ podem assumir os valores 1,2 , 3 e 48 . Matematicamente, tensores de posto 2 (dois índices, no caso, $\mu$ e $\nu$ ) são matrizes de números, ou de funções, que se transformam de acordo com certas regras sob uma mudança de coordenadas. Assim, na TRG, os tensores são representados através de matrizes $4 \times 4$.

$\mathrm{Na}$ física, os tensores caracterizam as propriedades de um dado sistema. Um tensor pode consistir de um único número, sendo neste caso chamado de tensor de posto zero ou, simplesmente, escalar. Exemplos de escalar são: massa de uma partícula, volume de um planeta, temperatura de uma estrela ...

Exemplos de campos escalares são a densidade de um fluido em função da posição. Outro exemplo é a energia potencial gravitacional em função da posição. Note que ambos, densidade e energia potencial gravitacional, são números únicos (funções) que variam continuamente de ponto a ponto, definindo assim um campo escalar.

O próximo tensor é o de posto um, também conhecido com o nome de vetor. No espaço tridimensional comum, um vetor tem três componentes (contém três números ou três funções da posição). No espaço-tempo de quatro dimensões, um vetor tem quatro componentes (três componentes espaciais e uma temporal).

Um vetor pode ser considerado uma matriz coluna

\footnotetext{
${ }^{8}$ Utilizarei nesta Seção a mesma notação dos trabalhos originais de Einstein.
} 


\section{CORE) ( (OES \\ CI ÊNCIAETECNOLOGIA}

AVANÇO DO PERIÉLIO DE MERCÚRIO - O PRIMEIRO SUCESSO DA TEORIA DA RELATIVIDADE GERAL DE EINSTEIN

ou linha, dependendo do arranjo de termos (a dimensão, então, seria $n \times 1$ ou $1 \times n$ ). Isso ocorre porque os componentes de um vetor podem ser visualizados como sendo escritos em uma coluna ou ao longo de uma linha.

Acima de um vetor estão os tensores de posto 2, que são organizados na forma de matrizes. Assim como os vetores representam propriedades físicas mais complexas do que os escalares, as matrizes representam propriedades físicas ainda mais complexas do que as que podem ser manipuladas por vetores 9 .

Voltando à TRG de Einstein, a curvatura do espaçotempo, que dá origem à gravidade, é descrita pelo tensor de Ricci. Este, por sua vez, é obtido a partir de um tensor de curvatura, mais geral, de posto 4 (quatro índices como, por exemplo, $\mu, \nu, \xi$ e $\beta$ ), que é chamado tensor de Riemann. A caracterização da curvatura do espaço-tempo é então obtida através do tensor $R_{\mu \nu}$ e de seu escalar $R$ na Equação 42.

Outro tensor presente nessa equação é o tensor métrico $g_{\mu \nu}$. Métrica significa um padrão de medida. Formalmente, o tensor métrico atua num espaço vetorial "recebendo" vetores e "retornando" números. É o objeto matemático que permite definir rigorosamente medidas de comprimento (ou de distância entre dois pontos) além de ângulos num dado espaço.

$\mathrm{Na}$ representação matricial, os números reais que descrevem os componentes desse tensor dependem do particular sistema de coordenadas que estiver sendo utilizado. Contudo, o tensor métrico sempre terá a propriedade de receber vetores e retornar números com úteis informações sobre a geometria do espaço em estudo.

Em termos de coordenadas, na notação usada por Einstein, o componente 4 representa a coordenada temporal do espaço-tempo. As três coordenadas espaciais são representadas como 1,2 e 3.

Se trabalharmos em coordenadas cartesianas, então na TRG, respectivamente, descrevem $x, y, z$ e $t$. No caso de coordenadas esféricas, descrevem $r, \theta, \phi$ e $t$. De forma geral, as coordenadas são representadas como $x_{1}, x_{2}, x_{3}$ e $x_{4}$ com a coordenada temporal sendo $x_{4}$.

Veja que o lado esquerdo da Equação 42 apresenta grandezas puramente geométricas, isto é, curvatura, medidas de distâncias e ângulos. Já do lado direito de 42 , nós temos o tensor $T_{\mu \nu}$ que descreve os campos de matéria e radiação presentes numa dada região do espaço-tempo.

Assim, os físicos dizem que as equações da TRG de Einstein, representadas em 42 , relacionam a geometria

\footnotetext{
${ }^{9}$ Procuramos neste trabalho introduzir o conceito de tensores de forma mais simples e natural. Aos leitores interessados em compreender o conceito de tensores com maior rigor matemático, sugiro o excelente texto de SYNGE e SCHILD (1978).
}

(lado esquerdo) com a física (lado direito). Isto é, matéria e radiação dizem ao espaço-tempo como se curvar; por outro lado, a curvatura diz às matéria e luz como elas devem se comportar.

A força gravitacional é o resultado desse acoplamento entre a curvatura do espaço-tempo e os campos de matéria e radiação que permitem a tudo descrever (planetas, estrelas, galáxias, partículas, luz ...) no Universo.

Em 1915, Einstein começou a determinar $g_{\mu \nu}$ para o Sol. O sistema solar pode ser encarado como uma massa isolada, que está longe de outras massas no Universo. Quase 99 por cento da massa total do sistema solar está concentrada no Sol.

Assim, podemos tratar os planetas como pontos de massa que se movem no campo gravitacional estático do Sol. Dentro do sistema solar, pode-se negligenciar o potencial gravitacional dos planetas e lidar apenas com o potencial gravitacional do Sol, considerando-o como uma estrela com simetria esférica.

Por causa da distância separando o planeta da massa central, a curvatura do espaço-tempo vai gradualmente diminuindo a partir do Sol, tornando-se plana a grandes distâncias. Essas foram as condições que Einstein impôs ao campo gravitacional do Sol.

A partir desse raciocínio, ele iniciou seu cálculo tomando as Equações 42 escritas para o vácuo (o que significa tomar $T_{\mu \nu}=0$ ), obtendo então

$$
\sum_{\alpha} \frac{\partial \Gamma_{\mu \nu}^{\alpha}}{\partial x_{\alpha}}+\sum_{\alpha \beta} \Gamma_{\mu \beta}^{\alpha} \Gamma_{\nu \alpha}^{\beta}=0
$$

onde $\Gamma_{\mu \nu}^{\alpha}$ são os componentes do campo gravitacional determinados através de

$$
\Gamma_{\mu \nu}^{\alpha}=-\frac{1}{2} \sum_{\beta} g^{\alpha \beta}\left(\frac{\partial g_{\mu \beta}}{\partial x_{\nu}}+\frac{\partial g_{\nu \beta}}{\partial x_{\mu}}-\frac{\partial g_{\mu \nu}}{\partial x_{\alpha}}\right)
$$

Adicionalmente, Einstein usou $\sqrt{-g}=1$ como condição para as coordenadas.

O lado esquerdo da 43 é o tensor de Ricci, que engloba o tensor métrico e suas derivadas. As Equações 43 são não lineares por causa de $\Gamma_{\mu \nu}^{\alpha}$.

Em seguida, ele partiu da chamada aproximação de ordem zero, em que $g_{\mu \nu}$ corresponde à teoria da relatividade especial (ou a chamada "métrica plana de Minkowski" ). Isto é,

$$
g_{\mu \nu}=\operatorname{diag}(-1,-1,-1,+1),
$$

onde "diag" significa que os únicos elementos não nulos são os que pertencem à diagonal da matriz de representação do tensor $g_{\mu \nu}$. Veja que $g_{11}=g_{22}=g_{33}=-1$ 


\section{CORE) ( (OES \\ CIÊNCIAE TECNOLOGIA}

AVANÇO DO PERIÉLIO DE MERCÚRIO - O PRIMEIRO SUCESSO DA TEORIA DA RELATIVIDADE GERAL DE EINSTEIN

são os componentes espacias do tensor métrico, enquanto $g_{44}=+1$ é o componente temporal.

Einstein escreveu a Equação 45 como

$$
g_{\rho \sigma}=\delta_{\rho \sigma}, \quad g_{\rho 4}=g_{4 \rho}=0, \quad g_{44}=1,
$$

onde os índices $\rho$ e $\sigma$ significam 1,2 e 3. O delta de Kronecker $\left(\delta_{\rho \sigma}\right)$ é igual a 1 se $\rho=\sigma$ e é igual a zero se $\rho \neq \sigma$.

A aproximação representada na Equação 46 é a aproximação de ordem zero. Einstein então assumiu que $g_{\mu \nu}$ difere dos valores dados na Equação 46 por uma pequena quantidade quando comparada a 1, tratando esse desvio como uma pequena mudança de "primeira ordem". A solução para o campo métrico $g_{\mu \nu}$ tem quatro propriedades, que implicam em quatro propriedades para o campo gravitacional do Sol:

- A solução é estática. Todos os componentes da solução são independentes da coordenada temporal $\left(x_{4}\right)$.

- A solução $g_{\mu \nu}$ é esfericamente simétrica em relação à origem do sistema de coordenadas.

- As equações $g_{\rho 4}=g_{4 \rho}=0$ são válidas para $\rho=$ $1,2,3$.

- No infinito, $g_{\mu \nu}$ tende aos valores da métrica plana de Minkowski da relatividade especial, como representado na Equação 46 .

Para primeira ordem, as quatro condições acima fornecem a seguinte solução

$$
g_{\rho \sigma}=-\delta_{\rho \sigma}-\alpha \frac{x_{\rho} x_{\sigma}}{r^{3}}, \quad g_{44}=1-\frac{\alpha}{r},
$$

onde $g_{\rho \sigma}$ tende à métrica de Minkowski, conforme Equação 46, através da quarta propriedade acima, enquanto $g_{\rho 4}$ e $g_{4 \rho}$ são determinados pela terceira propriedade.

É direto verificar que as quatro propriedades, para o campo gravitacional do Sol, são preservadas pela solução de primeira ordem representada em 47. Para isso, basta substituir a solução 47 nas Equações 43 e 44.

A quantidade $r$ é obtida como

$$
r=\sqrt{x_{1}^{2}+x_{2}^{2}+x_{3}^{2}},
$$

enquanto $\alpha$ é

$$
\alpha=\frac{2 G M}{c^{2}},
$$

\section{com $M$ sendo a massa do Sol.}

Posteriormente, Einstein obteve o valor para os componentes do campo gravitacional do Sol em segunda ordem. Ele escreveu as equações de movimento para uma massa pontual movendo-se sob ação do Sol. Um planeta em queda livre sob ação de um campo gravitacional move-se em uma linha geodésica $(s)$ de acordo com a equação (EINSTEIN, 1915a)

$$
\frac{d^{2} x_{\nu}}{d s^{2}}=\sum_{\sigma \tau} \Gamma_{\sigma \tau}^{\nu} \frac{d x_{\sigma}}{d s} \frac{d x_{\tau}}{d s} .
$$

A Equação 50 contém as equações Newtonianas de movimento como uma primeira aproximação. Assim, Einstein calculou as equações das linhas geodésicas e comparou-as com as equações Newtonianas das órbitas dos planetas no sistema solar. Ele verificou que havia correspondência entre a relatividade geral e a teoria de Newton, em que a atração gravitacional é uma força central e todos os planetas movem-se em um plano constante em torno do Sol (vide Seção 2]).

Portanto, nas coordenadas polares, o movimento sobre esse plano depende da distância $r$, do planeta ao centro de massa do sistema, e do ângulo formado entre a linha que conecta o planeta ao centro e uma linha de referência escolhida arbitrariamente (vide, em particular, a Figura 2). Assim procedendo, obtemos a equação da órbita planetária, cuja solução para o caso Newtoniano descreve uma elipse.

O periélio da órbita é o ponto em que o planeta está mais próximo do Sol. Como pode ser visto na Figura 3 o eixo principal da elipse, descrita por Mercúrio, gira lentamente em torno do Sol. Essa é a precessão do periélio, sendo mais pronunciada quanto maior for a excentricidade $e$ da órbita.

$\mathrm{Na}$ teoria de Einstein, a Equação 50 leva a uma equação relativística para as orbitas planetárias. Einstein descobriu que a diferença entre as equações orbitais (Newtoniana e relativística) estava em um termo adicional $2 G M / c^{2} r^{3}$.

Com essas aproximações, Einstein obteve a forma como a curvatura do espaço-tempo influencia o ângulo apsidal. Isto é,

$$
\Psi=\pi\left[1+\frac{3}{2} \frac{\alpha}{a\left(1-e^{2}\right)}\right] .
$$

Assim, a cada órbita o periélio avança

$$
\omega=2 \Psi-2 \pi=3 \pi \frac{\alpha}{a\left(1-e^{2}\right)} .
$$

Usando os valores para a massa do Sol $(M)$, o semieixo maior da órbita $(a)$ e excentricidade da órbita (e), torna-se possível reescrever a Equação 52 como 


\section{CORE) ( (OES \\ CIÊNCIA E TECNOLOGIA}

AVANÇO DO PERIÉLIO DE MERCÚRIO - O PRIMEIRO SUCESSO DA TEORIA DA RELATIVIDADE GERAL DE EINSTEIN

$$
\omega=\pi \times 1,60355 \times 10^{-7} .
$$

A taxa com que o periélio de Mercúrio precessiona é então

$$
\dot{\omega}=\frac{\omega}{P}=\frac{\pi\left(1,60355 \times 10^{-7}\right)}{87,969 \operatorname{dias}} .
$$

O resultado da contribuição de efeitos relativísticos gerais para $\dot{\omega}$ é, dessa forma,

$$
\dot{\omega}=43,1 " \text { por século. }
$$

Somando o previsto pela teoria Newtoniana (41) com a "anomalia" associada com a curvatura do espaçotempo 55, nós obtemos o valor $\dot{\omega}=575$ " por século para a precessão do periélio de Mercúrio. Valor que está em acordo com os dados observacionais.

Esse foi o primeiro triunfo da Teoria da Relatividade Geral. Em particular, Einstein concluiu seu trabalho de 1915 (EINSTEIN, 1915a) escrevendo:

"O cálculo produz, para o planeta Mercúrio, um avanço do periélio de 43" por século, enquanto os astrônomos indicaram 45" $\pm 5 "$ por século como uma diferença inexplicável entre as observações e a teoria Newtoniana. Esta teoria, portanto, concorda completamente com as observações" 10 .

\section{Considerações Finais}

Neste trabalho, cobrimos cerca de quatro mil anos de estudos sobre os movimentos planetários. Partindo da antiga Mesopotâmia cerca de 2.000 a.C., em que os movimentos planetários começaram a ser registrados de forma sistemática, passando pela contribuição dos gregos na construção dos primeiros modelos destinados a explicar o "funcionamento do sistema solar" , nós cobrimos vinte séculos de história. O modelo Geocêntrico, estruturado durante esse período, perpetuou-se até a idade média quando foi gradativamente sendo substituído pelo modelo Heliocêntrico.

Galileo Galilei e, em particular, Isaac Newton foram responsáveis por um gigantesco salto na compreensão do movimento planetário, bem como da estruturação da área do conhecimento que hoje chamamos de "física". Até o início de 1781, além da Terra, eram conhecidos os mesmos cinco planetas que os povos da Suméria estudavam e acompanhavam através de registros feitos em escrita cuneiforme. Nesse ano, William Herschel descobriu Urano de forma acidental.

\footnotetext{
${ }^{10}$ Aos interessados em seguir o raciocínio completo de Einstein, sobre a "anomalia" do periélio de Mercúrio, sugiro acessar o hiperlink da referência Einstein (1915a) na qual as notas desta Seção estão baseadas.
}

Com base na bem estabelecida teoria de gravitação Newtoniana, um novo salto no estudo do sistema solar foi dado por Urbain-Jean-Joseph Le Verrier que concentrou-se no cálculo preciso das órbitas planetárias. Le Verrier previu a existência de mais um planeta no sistema solar - Netuno - encontrado posteriormente no quadrante do céu que ele, matematicamente, havia identificado.

O sucesso alcançado pela teoria Newtoniana da gravitação esbarrou na observada "anomalia" de Mercúrio. Le Verrier desenvolveu um seminal trabalho matemático, mostrando que a órbita de Mercúrio lentamente precessionava por influência do campo gravitacional do Sol e dos demais planetas em órbitas externas à Mercúrio.

Ele obteve, a partir dos seus cálculos, o valor de 526,7 segundos de arco por século, enquanto a precessão observada era de 565 segundos de arco por século. Essa diferença de 38 " por século configurava-se numa "anomalia" que não podia ser explicada pela gravitação Newtoniana.

A partir do sucesso alcançado com a previsão do planeta Netuno, Le Verrier chegou a propor a existência de um novo planeta, chamado Vulcano, em órbita intermercurial. Com base nas observações do astrônomo amador Lescarbault, Le Verrier calculou a distância de Vulcano em relação ao Sol, obtendo 0,147 unidades astronômicas. O hipotético planeta possuiria período orbital de 19 dias e 17 horas.

Após diversas tentativas de observar Vulcano, a comunidade científica foi gradativamente deixando de acreditar na existência desse planeta. Simon Newcomb, em 1882, corrigiu algumas inconsistências na massa planetária e repetiu os cálculos de Le Verrier, decobrindo um deslocamento extra no periélio de Mercúrio de 43 " por século. Os dados observacionais, naquela época, mostravam que a precessão de Mercúrio era de, aproximadamente, 575 " por século.

Newcomb ponderou que se o expoente, na lei da gravitação de Newton, fosse 2,00000016 ao invés de 2, então o movimento de Mercúrio poderia ser explicado com maior precisão. Esse raciocínio configurouse numa mudança de paradigma. Anteriormente, as observações eram questionadas e a teoria de Newton era inatacável. A partir dessa análise de Newcomb, os cientistas ao final do século XIX começavam a questionar as fundações da lei de gravitação clássica.

Isso abriu caminho para que Einstein introduzisse, em 1915, uma das mais belas teorias da física - a Teoria da Relatividade Geral. A força gravitacional passa a ser tratada como uma resposta à curvatura do espaçotempo, tecido que permeia todo o Universo. No mesmo 


\section{CORE) ( (OES \\ CI ÊNCIAETECNOLOGIA}

AVANÇO DO PERIÉLIO DE MERCÚRIO - O PRIMEIRO SUCESSO DA TEORIA DA RELATIVIDADE GERAL DE EINSTEIN

artigo em que descreve a sua teoria de gravitação, Einstein mostra que a "anomalia" de 43 " por século, na precessão do periélio de Mercúrio, pode ser perfeitamente explicada com base na sua teoria.

No ano seguinte, Einstein submeteu ao periódico Annalen der Physik um artigo de revisão sobre a Teoria da Relatividade Geral (EINSTEIN, 1916) . Ele derivou a equação para a deflexão de um raio de luz vindo de uma estrela de fundo e passando próximo ao Sol.

Em função do sucesso da TRG em explicar a "anomalia" de Mercúrio, em 1915, e da previsão de que raios luminosos de estrelas distantes seriam curvados pelo Sol, feita em 1916, a comunidade científica preparouse para esse segundo teste da teoria de Einstein.

A confirmação dessa previsão foi obtida por ocasião do eclipse total do Sol ocorrido no dia 29 de maio de 1919, visível na Ilha de Príncipe (África) e em Sobral (Ceará - Brasil).

Em poucos anos, a nova teoria de gravitação encantou os cientistas por sua beleza conceitual e pelas previsões sucessivamente confirmadas através de observações e experimentos.

\section{REFERÊNCIAS}

BAUM, R.; SHEEHAN, W. In Search of Planet Vulcan: The Ghost in Newton's Clockwork Universe. New York: Springer and Plenum Press, 1997.

EINSTEIN, A. Erklärung der perihelbewegung des merkur aus der allgemeinen relativitätstheorie. Königlich Preußische Akademie der Wissenschaften, Sitzungsberichte, p. 831-839, 1915a. Disponível em: $<$ https://einsteinpapers.press.princeton.edu/vol6-trans/ $124>$

Zur allgemeinen relativitätstheorie. Königlich Preußische Akademie der Wissenschaften, Sitzungsberichte, p. 778-786, 1915b. Disponível em: $<$ https://einsteinpapers.press.princeton.edu/vol6-trans/ $110>$

Zur allgemeinen relativitätstheorie (nachtrag). Königlich Preußische Akademie der Wissenschaften, Sitzungsberichte, p. 799-801, 1915c. Disponível em: <https: //einsteinpapers.press.princeton.edu/vol6-trans/120>

Die grundlage der allgemeinen relativitätstheorie. Annalen der Physik, v. 49, p. 769-822, 1916. Disponível em: <https://einsteinpapers.press. princeton.edu/vol6-trans/158>.
EVANS, J. The History and Practice of Ancient Astronomy. New York and Oxford: Oxford University Press, 1998.

LAMBERT, W. Babylonian astrological omens and their stars. Journal of the American Oriental Society, American Oriental Society, v. 107, n. 1, p. 93-96, 1987. Disponível em: <https://www.jstor.org/stable/602955>

LASKAR, J. Des premiers travaux de le verrier à la découverte de neptune. Comptes Rendus Physique, v. 18, p. 504-519, 2017. Disponível em: <https://doi.org/10.1016/j.crhy.2017.10.011>

LE-VERRIER, U. J. J. Détermination nouvelle de l'orbite de mercure et de ses perturbations. Comptes Rendus de l'Académie des Sciences, v. 16, n. 19, p. 1054-1065, 1843a.

Tables de mercure. Comptes Rendus de l'Académie des Sciences, v. 16, n. 23, p. 1280, 1843 b.

LéVY, J. Trois siècles de mécanique céleste à l'observatoire de paris. L'Astronomie, v. 82, p. 381-393, 1968. Disponível em: <http://articles.adsabs. harvard.edu/full/1968LAstr..82..381L>

MIRANDA, O. Buracos negros. Revista Brasileira de Astronomia, v. 1, n. 1, p. 12-21, 2019. Disponível em: $<$ https://sab-astro.org.br/sab/rba/>

NEUGEBAUER, O. A History of Ancient Mathematical Astronomy. New York, Heidelberg, Berlin: Springer, 1975.

Astronomy and History Selected Essays. New York: Springer, 1983.

NEWCOMB, S. Discussion and results of observations on transits of mercury from 1677 to 1881 . Astronomical Papers American Ephemeris and Nautical Almanac, Washington, Bureau of Navigation, Navy Department, v. 1, n. 1, p. 363-487, 1882. Disponível em: <http: //adsabs.harvard.edu/abs/1882USNAO...1..363N>

Tables of the four inner planets. Astronomical Papers American Ephemeris and Nautical Almanac, Washington, Bureau of Navigation, Navy Department, v. 6, n. 3, p. 586, 1898. Disponível em: <http: //adsabs.harvard.edu/abs/1898USNAO...6Q...1N>

NEWTON, I. Principia - Livro I: Princípios matemáticos de filosofia natural. São Paulo: EDUSP, 2008a. 


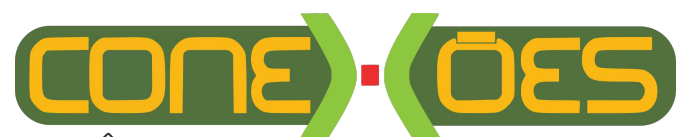

CIÊNCIA E TECNOLOGIA

AVANÇO DO PERIÉLIO DE MERCÚRIO - O PRIMEIRO SUCESSO DA TEORIA DA RELATIVIDADE GERAL DE EINSTEIN

. Principia - Livro II e III: Princípios matemáticos de filosofia natural. São Paulo: EDUSP, 2008b.

PRICE, M.; RUSH, W. Nonrelativistic contribution to mercury's perihelion precession. American Journal of Physics, v. 47, p. 531-534, 1979. Disponível em: $<$ https://aapt.scitation.org/doi/pdf/10.1119/1.11779? class $=$ pdf $>$.

ROGAWSKI, J.; COLIN, A. Calculus. New York:

W.H. Freeman \& Company, 2015.

SYNGE, J.; SCHILD, A. Tensor Calculus. New York:

Dover Books on Mathematics, 1978. 